\title{
Dénominations Polémiques, Mépris et contre Mépris dans les Discours Produits entre 2017-2019 sur les Réfugiés en Algérie
}

\author{
Kamila Oulebsir-Oukil * \\ LISODIP, ENS-Bouzaréah Algérie
}

Received on: 12-4-2020

Accepted on: 16-8-2020

Résumé

Dans cette contribution, nous souhaitons étudier l'expression du mépris tel qu'il est construit dans le discours médiatique concernant les réfugiés en Algérie. Nous voudrions montrer la façon dont les réfugiés sont dénommés et comment le discours émanant des ONG et d'autres associations internationales accuse l'Algérie de mépriser ses réfugiés. Il sera également question de voir comment ce pays construit sa réponse envers ces accusations de mépris. En effet, les réfugiés en Algérie sont fortement stigmatisés et vivent, par conséquent, dans la précarité. Notre étude tentera de déceler ce qui pourrait relever d'un sentiment de mépris envers ces personnes.

Mots-clés: Mépris, Acte de langage, Discours médiatique, Analyse du discours, Polémique.

\section{Polemical Denominations, Contempt and Counter-Contempt in the Speeches Produced between 2017-2019 on Refugees in Algeria}

\begin{abstract}
In this contribution, we study the expression of contempt as it is constructed in the media discourse concerning refugees in Algeria. We show the way in which discourse emanating from NGOs and other international associations accuses Algeria of despising its refugees. The study is also concerned with how this country constructs its response to these accusations of contempt. Refugees in Algeria are strongly stigmatized and therefore live in precarious conditions. Our study will try to identify what could be a feeling of contempt for these people.
\end{abstract}

Keywords: Contempt, Speech acts, Media discourse, Discourse analysis, Controversy.

\section{Introduction}

La question des réfugiés retient l'attention de plusieurs pays et occupe particulièrement les débats actuels en Algérie. C'est une question sensible qui interpelle le gouvernement algérien, mais aussi les citoyens qui accueillent ces personnes (associations, centres d'accueil et différentes organisations). D'après les témoignages que l'on peut lire dans les différents médias ${ }^{1}$, les réfugiés se qualifient de personnes qui sont confrontées à des conditions difficiles et inhumaines et qui subissent l'humiliation et le refus de se

\footnotetext{
๑ 2021 JJMLL Publishers/Yarmouk University. All Rights Reserved.

* Doi: https://doi.org/10.47012/jjmll.13.2.8

* Corresponding Author: kamilaoulebsir83@gmail.com
} 
faire reconnaitre un statut. Ces gens, principalement des Syriens, des Subsahariens et des Palestiniens, vivent malaisément dans les pays d'accueil, en l'occurrence l'Algérie.

La Convention de Genève du 28 juillet 1951 définit le statut de réfugié et renseigne sur les critères requis pour en bénéficier ${ }^{2}$. Ce document énumère les droits des réfugiés ainsi que leurs obligations envers les pays qui les accueillent. Le terme réfugié renvoie, par définition, à toute personne forcée de quitter son pays pour fuir les guerres, les différents conflits, les crises politiques, etc.

L'Algérie affirme la volonté de protéger les droits des personnes en mobilité, comme le stipule l'article 81 de la Constitution algérienne: «Tout étranger qui se trouve légalement sur le territoire national jouit, pour sa personne et pour ses biens, de la protection de la loi » ${ }^{3}$. Cependant, ce même texte ne reconnait pas l'accès au statut de réfugié:

La Constitution algérienne interdit déjà le renvoi forcé de réfugiés politiques (article 83) mais ne mentionne pas le droit de demander asile. Elle ne reconnait pas non plus

le doit d'échapper à la persécution et à d'autres formes de graves atteintes, conformément à la Convention de 1951 relative au statut des réfugiés ${ }^{4}$.

Dans cette contribution, nous voulons étudier quelques stratégies discursives à travers lesquelles le mépris dont les réfugiés sont victimes est mis en mots. Nous nous posons alors les questions suivantes : quelles sont les stratégies méprisantes et stigmatisantes mises en œuvre dans les discours évoquant les réfugiés ? Comment le traitement de l'Algérie envers les réfugiés est dénoncé par les ONG ? Quelle est la réaction de l'Algérie face à ces accusations de mépris ?

En Algérie, les discours tenus par les ONG et les associations défendant les réfugiés dénoncent les conditions dans lesquelles vivent ces personnes tout en qualifiant les actions et les comportements adoptés à leur égard. En effet, le traitement que réservent les autorités algériennes aux réfugiés est qualifié, par certaines ONG et associations ${ }^{5}$, de scandaleux, et ce, malgré les textes officiels censés appliquer les points stipulés par Convention de Genève. Face à ces accusations, l'Etat algérien répond, à travers ses sources officielles et les chiffres avancés, pour affirmer qu'il accueille sur son territoire des dizaines de milliers de réfugiés notamment les réfugiés issus de pays en guerre : Syriens, Libyens, Malien, Palestiniens et environ cent mille réfugiés du Sahara Occidental dans les camps de Tindouf ${ }^{6}$.

\section{2- Précisions théoriques}

L'étude s'inscrit dans le domaine de l'analyse du discours et propose une lecture contextualisée du corpus. Plus précisément, nous privilégions une approche pragmatique puisqu'il est question d'analyser les actes de langage à travers lesquels se construit le mépris envers les réfugiés. Nous procèderons également au repérage des marqueurs énonciatifs qui permettent de commenter les discours produits par différentes instances énonciatives, en l'occurrence celles qui sont responsables du dossier des réfugiés en Algérie, pour ainsi asseoir leur position face à la problématique des réfugiés.

Avant de présenter le cadre théorique, nous faisons deux remarques importantes pour notre étude. Ces deux idées sont reliées et définissent au mieux l'analyse de notre corpus : 
Dénominations Polémiques, Mépris et contre Mépris dans les Discours

Produits entre 2017-2019 sur les Réfugiés en Algérie

1- Nous travaillons d'emblée sur des discours rapportés par différentes sources énonciatives qui accusent l'Algérie de maltraiter les réfugiés. Nous analysons, à la fin de l'article, la position officielle de l'Algérie, en l'occurrence celle rapportée par la Présidente du Croissant Rouge, pour répondre à ces accusations de mépris. Nous nous basons sur le discours rapporté comme point de saisie de notre corpus pour repérer le mépris envers les réfugiés dont est accusée l'Algérie.

2- Nous analysons la construction indirecte du mépris telle qu'elle se déploie à travers les dénominations et les qualifications du traitement que l'Algérie réserve aux réfugiés. En termes plus précis, le mépris dont il sera question dans les analyses ci-dessous est perçu dans le comportement que ce pays adopte face aux réfugiés. Les ONG et les autres associations condamnent le comportement de l'Algérie et montrent, par ricochet, le mépris de ce pays envers les réfugiés.

En somme, le mépris est appréhendé, dans notre corpus, à travers deux axes. Le premier est déduit de l'acte d'accusation et de condamnation du comportement de l'Algérie face aux réfugiés qui est jugé, par les ONG, comme inopportun et inacceptable. Le second est centré sur les dénominations données aux réfugiés et les qualifications péjoratives utilisées à leur égard et qui sont rapportées par le discours médiatique.

\subsection{Des actes de langage à la violence verbale}

Le mépris et sa mise en mots et en discours font l'objet de peu d'études, notamment dans le domaine des sciences du langage. Nous proposons une façon d'analyser le mépris tel qu'il est défini en tant qu'acte de langage dépréciatif indirect (Moïse, 2012). Cet acte a une valeur perlocutoire précise, celle de catégoriser l'autre et de le rabaisser en raison du statut de dominé qui lui est accordé par celui ou celle qui le méprise (Larguèche, 2011). Le mépris peut surgir dans un discours tenu pour qualifier et stigmatiser l'action ou les propos de l'autre, forcément différent de soi. Dans ce sens, il est possible de classer le mépris dans « les actes de condamnation du faire » et « de l'être » (Laforest et Moïse, 2013), considérés comme des actes de langage à travers lesquels s'exprime une insatisfaction conduisant au jugement d'un propos, d'un acte ou d'un être.

Défini comme un acte de langage dépréciatif et menaçant (Laforest et Moïse, 2013 ; Moïse et SchulzRomain, 2011), et comme une des étapes de la violence verbale fulgurante (Moïse 2012), le mépris traduit un sentiment de refus de l'autre, de déni, d'indifférence et de dégout. En effet, ces auteurs confirment que le mépris est un acte à travers lequel peut se décliner une violence verbale. Nous nous contentons, dans cette section, d'une brève précision concernant le rapport entre les actes de langage, l'insulte et le mépris. Notre travail s'inscrit dans le cadre selon lequel le langage est une action, en référence à la conception d'Austin (1969) et Searle (1969). En effet, les actes de langage, définis dans le cadre de la pragmatique et des théories des actes de langage sont pris comme action et moyen d'agir sur autrui et sur le monde. Les énoncés n'ont pas une valeur de vérité mais une valeur d'action. Cette valeur n'est pas toujours explicitement exprimée. Le sens des actes ne préexiste pas au discours mais il est construit en filigrane au moment de leur production. Le récepteur d'un message, d'un acte, est à même d'interpréter et de conclure à une condamnation, une insulte ou un mépris. 
Le mépris est un acte de langage dépréciatif généré par un autre acte de langage direct sous-jacent que nous tenterons de définir au moment de l'analyse. Pour réaliser cet acte, l'acte de langage direct subit un processus de dérivation illocutoire (Kerbrat-Orecchioni 2005, Anscombre 1977) c'est-à-dire que sa première visée est dérivée. L'un des effets produits sur le destinataire peut relever d'une attitude méprisante décelée dans le fait de refuser, de rabaisser ou de dominer l'autre. Le destinataire peut ressentir du mépris de la part d'autrui suite à un comportement ou à des paroles jugées dégradantes (Moïse 2012). A titre d'exemple, une insulte, un déni ou encore un harcèlement peuvent avoir pour fin l'expression d'un mépris. Le mépris est le résultat de cet état de dépréciation qui accompagne les propos d'un locuteur sur un thème ou une personne quelconque.

\subsection{Le mépris comme acte de langage}

Le mépris est un acte indirect de qualification péjorative constitutif de la violence verbale et caractérisé par la disqualification et la condamnation de l'autre. La nature de la relation existant entre les individus, en l'occurrence celle bâtie sur une différence de statut, peut expliquer le recours au mépris. Cette discrimination est une catégorisation de l'interlocuteur dans une classe inférieure à celle du locuteur qui se considère alors comme un «être au dessus» (Koselak, 2005, 7). En effet, Larguèche affirme que le fait d'être détenteur d'une position supérieure n'a pas en soi de caractère blessant, certaines activités entraînent de par leur nature des statuts de dominants et des statuts de dominés. Mais la position de dominant produit facilement des comportements méprisants à l'égard de ceux qui sont d'un statut inférieur et par conséquent dans une position de dominés $(2011,145)$.

Toujours incorporés aux actes menaçants, les actes de condamnation d'autrui, peuvent avoir des réactions et des réalisations identiques. Laforest et Moïse (2013) distinguent alors entre deux grandes catégories d'actes de condamnation. Il s'agit de la condamnation du faire qui consiste à exprimer une insatisfaction à propos d'un acte ou d'un comportement d'un individu que le locuteur juge inadéquat. La condamnation de l'être consiste à exprimer l'insatisfaction à l'égard d'une caractéristique d'un individu. Nous montrerons, dans cette étude, que les ONG accusent, à travers une condamnation du faire, l'Algérie de maltraiter et de mépriser les réfugiées.

Si le mépris est défini comme un acte de langage menaçant et que les actes de langage menaçants participent de la violence verbale (Auger, Fracchiolla, Moïse et Schulz-Romain, 2008), il y aura un lien entre la violence et le mépris. En effet, Moïse (2012) considère la violence fulgurante, qui est un type de violence verbale, comme une montée en tension contextualisée. Cette dernière est perçue à travers différentes étapes qui vont de l'incompréhension, de la négociation jusqu'à l'évitement ou le renchérissement. Ces étapes sont marquées par des déclencheurs de conflit ou des marqueurs discursifs qui signifient une rupture en termes de durcisseurs, de mots du discours ou d'effets syntaxiques. Elles peuvent aussi être marquées par des actes de langage dépréciatifs directs tels que le mépris, la provocation ou l'insulte à visée de domination. 
Dénominations Polémiques, Mépris et contre Mépris dans les Discours

Produits entre 2017-2019 sur les Réfugiés en Algérie

Sans pour autant affirmer que le mépris est insultant, nous rejoignons Moïse (2012) lorsqu'elle définit le mépris comme une résultante de l'insulte. Cette dernière est considérée comme un acte qui vise à faire ressentir un état affectif négatif chez l'interlocuteur et ce, par le recours à un discours portant atteinte à son identité. L'intention de cet acte est de rabaisser, de mépriser et de dégrader le destinataire.

Le mépris se manifeste concrètement par des qualifications et des connotations péjoratives transmises à travers des mots et des formules traduisant, dans quelques cas, des actes discriminatoires (Larguèche, 2011) basés sur le rejet et les stéréotypes produits sur l'autre qui est catégorisé dans une classe inférieure à celle du locuteur. Le ton peut également traduire un sentiment de mépris même si l'on prononce des mots dénués de cette dimension méprisante (Laforest et Moïse, 2013 ; Larguèche, 2011). Le silence et le refus de répondre peuvent aussi exprimer une attitude de mépris envers l'autre, considéré comme inférieur à celui qui s'exprime. Ce comportement conduit à donner une image dévalorisante du méprisé, à le discriminer et à le rejeter (Larguèche, 2011). Dans notre cas, nous rappelons que les discours que nous analysons condamnent le comportement et les décisions de l'Algérie face aux réfugiés, c'est ce qui pourrait signifier que ce pays les méprise.

\section{Corpus et méthodologie d'analyse}

Nous proposons une analyse des discours circulant dans la presse algérienne et étrangère publiés en ligne et concernant la question des réfugiés en Algérie. A cet effet, notre hypothèse de recherche est que le discours tenu sur les réfugiés en Algérie, rapporté par les médias locaux ou étrangers, est considéré comme méprisant par les ONG et les associations de défense des droits de l'Homme. Dans un premier temps, il s'agit donc d'étudier la mise en mots du mépris dans le discours rapporté par les médias. Dans un deuxième temps, nous proposons de revenir sur la construction d'un discours de réponse, celui tenu officiellement en Algérie et qui refuse ces accusations de mépris.

Notre corpus est construit autour de données hétérogènes (Garric et Longhi, 2012) et composé des sous-corpus suivants ${ }^{7}$ :

- Discours de presse publiés en ligne entre 2017 et 2019 : articles intégraux parus dans la presse algérienne

d'expression française et dans la presse étrangère.

- Articles publiés, dans la même période, sur des sites d'information algériens et africains.

Le choix de ces médias se justifie par le fait qu'ils ont traité la question des réfugiés en abordant leurs conditions de vie ainsi que les traitements reçus dans les différents pays d'accueil.

Pour constituer notre corpus, nous avons effectué une recherche par mots-clés sur les sites de journaux et les sites d'informations indiqués. Nous avons isolé tous les articles qui traitaient du thème des réfugiés. Les deux critères qui délimitent le corpus sont relatifs à la date de publication de ces textes et au thème en question. En effet, nous avons choisi les articles qui abordent le mépris exprimé envers les réfugiés. Ces articles sont parus entre 2017 et 2019. Nous justifions cette périodicité par le fait que le thème des réfugiés a commencé à être fortement glosé dans l'espace social algérien ces dernières années avec le flux important de ces personnes fuyant leur pays en guerre ou en crise. Finalement, le corpus est constitué d'un ensemble de 60 articles et passages de sites d'information comprenant 54732 mots. Nous tenons à signaler que nous 
nous écartons d'une étude quantitative et nous proposons une analyse qui se veut qualitative en ce sens qu'elle cible les mécanismes employés dans l'expression du mépris. Nous nous basons dans la sélection des discours sur ceux qui ont dénoncé le mépris exprimé envers les réfugiés. Cette sélection s'est faite à travers les qualifications, les dénominations, les champs lexicaux utilisés susceptibles de révéler un tel sentiment. Pour analyser le corpus, nous avons suivi une approche inductive. Ainsi, la lecture des discours dont nous disposons nous a permis de dégager les axes d'analyse que nous proposons ci-après. Nous avons choisi des bornes chronologiques allant de 2017 à 2019 pour justifier d'une hétérogénéité des données et pouvoir ainsi analyser plusieurs stratégies de la mise en mots du mépris. De plus, c'est à partir de l'été 2017 que des hashtags ${ }^{8}$ stigmatisant la présence de ces personnes en Algérie apparaissent sur les réseaux sociaux.

Sur le plan méthodologique et comme nous l'avons signalé auparavant, nous approchons notre corpus en combinant des approches, des théories et des concepts différents. Ainsi, nous investissons les outils de l'analyse du discours (Maingueneau 2014 et 2012) et de la pragmatique (Austin 1962 et Searle 1969) pour relever la dimension du mépris véhiculée dans ces discours. Egalement, nous faisons appel à divers concepts tels que les dénominations en discours (Calabrese 2015), les dénominations polémiques (Krieg-Planque 2009), et à plusieurs théories telles que la théorie des actes de langage (Austin 1962 et Searle 1969) celle de la violence verbale (Laforest et Moïse 2013) et celle de l'énonciation avec les travaux de Autier-Revuz 1995 sur la modalisation autonymique et ceux de Kerbrat-Orecchioni, (1980) pour l'étude des axiologiques.

Il sera question de repérer les lieux où le mépris est exprimé tant sur le plan linguistique que discursif. Selon notre interprétation, il apparait que la vague d'accusations de la part des ONG qui condamnent l'Algérie en matière de traitement inhumain réservé à ces personnes montre que ce pays les méprise. Nous essayerons de montrer le lien entre la formulation du mépris et cette série d'accusations qui ont accablé l'Algérie. Pour terminer, nous analysons la réaction, notamment celle de la Présidente du Croissant rouge algérien, face à ces accusations de mépris, une réaction où se conjugue un positionnement social et une légitimité énonciative pour mieux éclairer la question.

\section{Analyse du corpus}

Nous articulons notre analyse autour de deux discours. Le premier est un discours dénonciateur du mépris de l'État algérien : il met en avant les stratégies discursives à travers lesquelles le mépris se manifeste. Le mépris exprimé envers les réfugiés est rapporté à partir des accusations adressées à l'Algérie en matière de traitement des réfugiés. Il s'actualise à travers certaines dénominations ainsi que dans/par les commentaires métadiscursifs qui les accompagnent. Le deuxième discours, que nous qualifions de discours de réponse, est centré sur le refus de ce mépris, l'Algérie s'y oppose et refuse d'être accusée de mépris envers les réfugiés.

\subsection{Processus de (dis) qualification et mépris}

Les exemples ci-dessous reprennent les qualifications utilisées par les acteurs sociaux :

1- Ses propos sont qualifiés de «choquants et scandaleux » et sont considérés comme « dignes de l'extrême

droite européenne». Les défenseurs des droits de l'Homme estiment qu'un tel discours ne peut 
Dénominations Polémiques, Mépris et contre Mépris dans les Discours Produits entre 2017-2019 sur les Réfugiés en Algérie

qu'alimenter «le racisme». (https://www.liberte-algerie.com/actualite/migrants-le-derapagedouyahia-273230).

2- Le refoulement unilatéral des personnes en situation irrégulière laisse à penser que la société algérienne serait xénophobe. Mais pourquoi ce racisme anti-africain en Algérie? (...) Des questions s'imposent alors : pourquoi ce passage à l'acte très brutal des Algériens ? Ces derniers ont-ils perdu leur sens d'hospitalité? Détestent-ils vraiment les étrangers? Sont-ils atteints par le «complexe du noir»? (https://www.lematindz.net/news/20893-mais-pourquoi-ce-racisme-anti-africain-en-algerie.html)

Les substantifs et les adjectifs évaluatifs axiologiques Refoulement unilatéral, xénophobe, racisme, racisme anti-africain et complexe construisent un champ lexical mobilisé autour du dénigrement affiché envers les réfugiés venus en Algérie. Ces vocables sont intrinsèquement axiologiques et marqués «d'un trait de (dé) valorisation attaché au sémème de l'unité» (Kerbrat-Orecchioni 1980, 87). Dans les exemples 1 et 2 , nous remarquons le déploiement de plusieurs mécanismes qui montrent le soubassement d'un acte de mépris envers les réfugiés. Ainsi, dans l'exemple 1, ce sont les propos d'Ouyahia, ancien ministre algérien, qui ont été qualifiés avec les adjectifs affectifs choquants et scandaleux dénotant une réaction émotionnelle et un jugement de valeur de la part de l'énonciateur suite à ces propos parus dans l'article « source de crime de drogue et de plusieurs autres fléaux » (TSA-Algérie 2017) qui évoque les réfugiés lors d'une entrevue avec la presse. A la suite du classement de Kerbrat-Orecchioni (1980), ces deux adjectifs peuvent être considérés comme axiologico-affectifs dans la mesure où l'énonciateur manifeste une prise de position à l'encontre de ces propos et du comportement qui en résulte. Nous pensons que dans les limites de ce corpus, le mépris n'apparait pas de façon directe mais il est tributaire d'autres actions et sentiments (accusations, maltraitance). Ces propos sont comparés à ceux de l'extrême droite européenne en raison de leur caractère raciste et xénophobe. C'est à travers le verbe d'opinion estimer qu'est rapportée l'appréhension intellectuelle (Kerbrat-Orecchioni 1980) des défenseurs des droits de l'Homme vis-à-vis de leur position face à la question des réfugiés. De plus et à travers le procédé de la modalisation autonymique d'emprunt, défini comme l'insertion d'un discours autre dans un discours en train de se tenir (AuthierRevuz 1992, 1995), l'énonciateur rapporte les propos d'Ouyahia et signale ainsi une hétérogénéité énonciative. En effet, les guillemets accompagnant les vocables racisme, complexe du noir signalent une non-coïncidence du discours à lui-même, ces passages convergent vers un contenu raciste et un comportement dénoncé envers les réfugiés. Dans l'exemple 2, l'acte illocutoire porté par une série d'interrogations, notamment à travers le verbe évaluatif détester, vise à montrer l'étonnement et l'indignation face à l'accueil que l'Algérie réserve aux réfugiés. En effet, à l'aide des modalisateurs très et vraiment, l'énonciateur fait remarquer un décalage entre l'image de l'Algérie en tant que pays accueillant et le refoulement dont témoignent les réfugiés. Les vocables injurieux xénophobe, racisme et complexe du noir concrétisent cette catégorisation des réfugiés sur la base de la couleur de la peau. Les sous-entendus déduits de ces exemples montrent que l'Algérie est hostile envers les réfugiés, c'est ce que dénoncent fermement ces instances énonciatives.

C'est aussi à travers les stéréotypes que la xénophobie caractérisant l'accueil et le traitement des réfugiés est mise en avant, comme le montre l'exemple ci-dessous : 
3- Une prise de conscience des dérapages xénophobes ? (...) Dans le compte-rendu d'une table ronde à laquelle avaient été invités des ressortissants noirs africains vivant à Alger « Algérie africaine ou pas ? », El Watan du 3 juillet 2009, le journaliste établit la réalité de ce racisme : «Kahlouch, nigrou,

Babay, esclave (...)» (huffpostmaghreb.com/ yassin-temlali/ sur-le- migrant-subsaharie_b_ 5439191.html)

Ainsi, kahlouch ${ }^{9}$, qui signifie nègre en arabe algérien, nigrou (qui renvoie aussi à nègre), babay et esclave sont des termes désignant les personnes de couleur noire. Cet exemple dénonce le mépris envers les réfugiés à travers la mise en évidence des stéréotypes racistes stigmatisant ces personnes. C'est à travers le sémantisme des stéréotypes, employés pour évoquer le profil des réfugiés, que se construit l'acte du mépris (Ukhova 2020). Pour que le lexique employé soit méprisant et qu'il puisse être ressenti comme tel et provoquer un effet pragmatique, il faut que le destinataire le reçoive en tant que tel et ce, en s'appuyant sur l'évaluation des représentations sociales et des stéréotypes (Ukhova 2020). L'emploi de Kahlouch, nigrou, Babay pour parler des réfugiés connote et renforce le racisme et contribue à définir un propos méprisant. L'objet du mépris relève donc d'une catégorie de personnes jugées moins importantes ou mauvaises par rapport au locuteur, ce qui impliquerait l'exclusion de cette dernière. Pour Kerbrat Orechioni, «le trait axiologique est une propriété sémantique de certaines unités lexicales, qui leur permet dans certaines circonstances de fonctionner pragmatiquement comme des injures (...)». (1980, 76, c'est l'auteure qui souligne).

Ainsi, les propos tenus envers les réfugiés et les qualifications qui sont utilisées montrent une dimension de mépris sur deux plans. Le premier concerne les termes utilisés qui auraient l'intention de porter atteinte à l'identité des réfugiés. Le second porte sur les effets qui seraient ressentis par les réfugiés en tant qu'êtres dominés. Ainsi, la construction d'un discours ayant pour cible l'identité et le statut d'un être dominé et l'éventuel sentiment perçu allant dans ce sens participent de la définition et du déploiement du mépris en discours.

\subsection{Le mépris à travers les dénominations}

Le mépris exprimé à l'égard des réfugiés peut être approché au moyen des dénominations utilisées par les acteurs sociaux lorsqu'il est question de nommer ou de catégoriser ces personnes. Dans les exemples ci-dessous, les dénominations renvoient d'un côté au profil des réfugiés, décrits comme des chabihas, milices, déplacés, et, d'un autre côté, aux actions qui seraient commises par l'Algérie et dont elle est victime vu sa politique adoptée envers les réfugiés.

Nous verrons que les dénominations utilisées sont elles-mêmes porteuses d'enjeux et transmettent la position de ceux qui les utilisent car

Dénommer, c'est choisir au sein d'un paradigme dénominatif ; c'est faire "tomber sous le sens", c'est orienter dans une certaine direction analytique, l'objet référentiel ; c'est abstraire et généraliser, c'est classifier et sélectionner: l'opération dénominative, (...) n'est donc jamais innocente, et toute désignation est nécessairement «tendancieuse ». (Kerbrat_Orecchioni 1980, 119). 
Dénominations Polémiques, Mépris et contre Mépris dans les Discours

Produits entre 2017-2019 sur les Réfugiés en Algérie

4- L'accusation est autant étonnante qu'intrigante : des «chabihas » (fantômes), ces milices civiles à la solde du régime de Bachar al-Assad, mais en petit nombre, figurent parmi les réfugiés syriens arrivés en Algérie depuis quelques semaines. Selon Debbah El-Bakar Mohamed Nidal, les «déplacés », un vocable qu'il préfère au mot « réfugiés » les Syriens présents en Algérie sont divisés en cinq catégories (...) (Liberté, 15/08/2017).

Dans l'exemple 4, le lexique utilisé est méprisant par son sémantisme. Le terme chabihah $^{10}$ véhicule, à l'origine, une connotation dévalorisante. Utiliser ce vocable pour désigner les réfugiés en Algérie témoigne de cette catégorisation de cet Autre tout en le dévalorisant. Sur un autre plan, l'énonciateur, identifié comme un membre du collectif de soutien au peuple syrien, prend la défense des réfugiés qu'il nomme autrement, à savoir les déplacés, une dénomination généralisante (Kerbrat-Orrechioni 1980) qui serait moins stigmatisante. Une négociation du dire qui montre la position de cet énonciateur envers la catégorie des réfugiés syriens signalant une hétérogénéité dans laquelle s’inscrivent ses propos. Le vocable refugié, de surcroit abandonné au profit du vocable déplacés, trouve son plein sens, celui d'un vocable porteur de mépris et de connotation négative. Il y aurait un conflit dénominatif (Calabrese, 2015) qui découle d'un sentiment de décalage entre les mots et le réel. Cet énonciateur, qui jouit d'une légitimité énonciative puisqu'il est le porte-parole d'un collectif de soutien, attire l'attention sur le sort de ces personnes qu'on a déplacées de force. Il pointe du doigt le fait qu'elles sont démunies de leurs droits et qu'elles ne possèdent aucun statut. Leur donner le nom de réfugiés, c'est reconnaitre leur appartenance et une identité comme stipulé dans les accords internationaux entre les pays censés protéger les réfugiés. Or, ces personnes n'ont connu en réalité que le déplacement et non la reconnaissance d'un statut particulier. Ce représentant des réfugiés syriens en Algérie s'approprie l'acte de nommer ces personnes en remettant en cause une dénomination communément partagée.

Les exemples qui suivent réemploient des dénominations polémiques que les énonciateurs se réapproprient pour afficher leur position, car l'acte de nommer signifie prendre position et choisir certains sens inscrits dans un interdiscours (Calabrese 2015). Ces dénominations sont polémiques parce qu'elles engagent un débat public sur le contenu qu'elles véhiculent et sont porteuses d'un sens controversé :

5- L'argument du vide juridique concernant le statut des réfugiés et des demandeurs d'asile ne peut expliquer et encore moins justifier cette politique discriminatoire (https://maghrebemergent.info/lesmigrants-africains-en-algerie-victimes-d-un-racisme-d-etat-contribution/)

6- La FIDH dénonce la «chasse aux migrants» et Amnesty le «profilage ethnique ». (https://maghrebemergent.info/les-migrants-africains-en-algerie-victimes-d-un-racisme-d-etatcontribution/)

7- Malgré les protestations des ONG et les «préoccupations » exprimées par le ministre nigérien des Affaires étrangères, les autorités algériennes n'ont donné aucune explication. Au-delà des expulsions, c'est la méthode qui choque. Les associations dénoncent un "profilage racial». (https://www.jeuneafrique.com/490400/politique/en-algerie-les-travailleurs-migrants-se-cachentpour-survivre/) 
Dans l'exemple 5, l'énonciateur insiste sur le fait que la discrimination des réfugiés en Algérie ne se justifie pas même dans le cas d'un vide juridique concernant leur statut. Pour ce faire, il utilise la négation (ne peut expliquer et encore moins) et la dénomination politique discriminatoire qui est ici reprise sans marqueurs métadiscursifs, puisqu'il ne s'agit pas d'un discours rapporté mais probablement d'un ilot textuel employé sans guillemets pour décrire la politique de l'Algérie. Cependant, en 6 et 7, les énonciateurs emploient les passages entre guillemets comme un ilot textuel marqué par des guillemets et le fragment guillemeté est attribué à l'énonciateur qui l'a proféré (FDH, Amnesty et les associations), et est utilisé par ces énonciateurs qui le mettent en relief. Les dénominations utilisées dans les exemples ci-dessus témoignent de l'importante accusation adressée à l'Algérie en matière de politique adoptée face aux réfugiés. Ainsi, politique discriminatoire, chasse aux migrants, profilage ethnique et profilage racial renvoient à cette volonté de nommer l'action et d'expliquer ce à quoi les réfugiés font face en Algérie. Ces dénominations sont polémiques et sont considérées comme des formules (Krieg-Planque, 2003, 2009). En effet, les syntagmes politique discriminatoire, profilage ethnique et profilage racial ont fonctionné dans le discours médiatique relatif au contexte de la guerre yougoslave des années 1990. Ils ont la capacité de décrire la réflexion de cette époque et renvoient à des enjeux socio-politiques particuliers. Le syntagme chasse aux migrants $^{11}$, apparu en Algérie à l'été 2017, est aussi considéré comme formule reflétant la position officielle de l'Algérie face à la question des réfugiés. Ces quatre syntagmes sont donc des dénominations polémiques par rapport au conflit qu'elles suscitent et aux enjeux qu'elles sous-tendent. L'emploi autonymique des dénominations utilisées avec les guillemets les place sur le terrain d'une hétérogénéité montrée, puisque les énonciateurs empruntent ces syntagmes à d'autres instances, aux ONG et aux fédérations internationales défendant les droits de l'Homme ayant signalé et condamné cette maltraitance des réfugiés. Par politique discriminatoire, chasse aux migrants et profilage éthique, on affiche la position politique de l'Algérie qui va à l'encontre de ce qui est effectivement stipulé dans les rapports politico-diplomatiques entre l'Algérie et les pays africains. C'est une politique qui va amener l'Algérie à entreprendre des actions qualifiées de racistes et de xénophobes sur son territoire.

Nous pouvons enfin dire que l'utilisation de ces dénominations dans les discours qui abordent le sujet des réfugiés en Algérie accentue le mépris ressenti envers eux. Ces discours qui, à la fois, dénoncent les dénominations des réfugiés et des lexèmes qui servent à rabaisser autrui sur le plan social (chabihas, déplacés...) et des actions entreprises envers eux (profilage, chasse, politique disciminatoire...) mettraient en relation l'utilisation de ces dernières avec le mépris dont l'Etat algérien est accusé. Mais comment se structure le discours méprisant les réfugiés ? Quelles catégorisations sont-elles utilisées pour parler d'eux avec mépris ? C'est à ces questions que nous répondrons dans la section suivante.

\subsection{Catégorisations sous-tendant le mépris}

Le comportement envers les réfugiés est explicitement qualifié de méprisant dans les exemples cidessous :

8- Il semble bien que le gouvernement algérien agit ici avec l'assurance de l'impunité démontrant à maintes occasions son mépris des migrants et réfugiés en provenance d'Afrique subsaharienne. (...). Les 
Dénominations Polémiques, Mépris et contre Mépris dans les Discours

Produits entre 2017-2019 sur les Réfugiés en Algérie

textes régionaux et internationaux, pourtant signés et ratifiés, subissent le même sort de déni ou de mépris. (https://maghrebemergent.info/les-migrants-africains-en-algerie-victimes-d-un-racisme-detat-contribution/)

9- Les réfugiés africains sont parfois la cible d'actes racistes en Algérie (...) nuées humaines misérables qui « envahissent » les villes du nord du pays ! (...) Si cette vague d'Africanophobie grossit, c'est qu'il

y a derrière le préjugé, le stéréotype, l'intox, le dénigrement, la manipulation, etc. (https://www.lematindz.net/news/20893-mais-pourquoi-ce-racisme-anti-africain-en-algerie.html)

Les exemples ci-dessous parlent explicitement de mépris à travers les accusations adressées au gouvernement algérien. Cette fois-ci, le mépris qualifie le comportement de l'Algérie (exemple 8) ou il est interprété comme tel (exemple 9). A travers des vocables tels que mépris, déni, raciste et des dénominations à caractère racial (africanophobie) et des constructions qualificatives (qui envahissent), le discours construit dans ces passages explique l'acte brutal qui caractérise les relations Algériens-réfugiés et détermine les causes du traitement pour le moins choquant réservé à ces personnes. Les axiologiques raciste (exemple 9), méprisant et dénigrant (exemple 8) sont employés pour condamner les Algériens et dénoncer leur racisme envers les réfugiés, c'est ce qui identifie et actualise l'acte de mépris. Signifiant une attitude intellectuelle, la tournure impersonnelle subjective il semble que (exemple 8) comme modalité de l'éventualité ne prend pas en charge le point de vue de l'énonciateur mais il s'en sert pour convaincre le destinataire du mépris affiché à l'égard des réfugiés et de la responsabilité du gouvernement algérien. Les marqueurs linguistiques à charge axiologique et porteurs de valeurs dévalorisantes participent à la définition de l'acte de mépris.

Que ce soit d'une manière explicite à travers l'utilisation des vocables mépris et déni ou à l'aide de marqueurs énonciatifs divers tels que l'ilot textuel avec l'occurrence guillemetée « envahissent » empruntée à une autre source énonciative non identifiée, les exemples ci-dessus vont au fondement du mépris et le mettent en mots. Les guillemets qui accompagnent le verbe envahissent pourraient signifier une prise de distance ou une dénonciation de la part du journaliste-énonciateur en matière de comportement réservé aux réfugiés.

Néanmoins, à côté de ce discours méprisant le réfugié, exprimé à travers des mécanismes linguistiques et discursifs divers, l'Algérie répond en construisant, à travers des sources énonciatives bien identifiées et d'une certaine légitimité institutionnelle, son discours contre le mépris. Ce pays refuse les accusations dont il est l'objet.

\section{Construction d'un discours de réponse : l'Algérie face aux accusations}

Il s'agit, par l'analyse de ce discours de réponse, de montrer la mise en mots de cette attitude refusant l'accusation de mépris à l'égard des réfugiés en Algérie. Cette section de notre analyse montre comment les accusations de mépris sont réfutées par l'Algérie.

En effet, dans les exemples ci-dessous, il sera question d'étudier la dimension dialogique qui caractérise ces discours. Le discours tenu officiellement par l'Algérie interfère avec celui tenu par ceux qui l'accusent de mépriser les réfugiés : 


\subsection{Les opérations d'autonymie : les accusations ne correspondent pas à la réalité}

10- Réagissant aux accusations sur l' « abandon » de migrants dans le désert ou de la « maltraitance » dont ils seraient victimes, Hassen Kacimi, président du comité interministériel chargé de l'étude et du suivi des problèmes migratoires, affirme que les coupables sont tout indiqués: les passeurs. (https://www.elwatan.com/edition/actualite/les-ong-plaident-pour-un-cadre-legal-dans-la-gestiondes-migrants-les-operations-de-rapatriement-au-compte-gouttes-16-09-2018)

11- Les autorités algériennes semblent agacées par l'appel des défenseurs des droits de l'Homme dénonçant le traitement infligé aux migrants subsahariens, dont pas moins de 1500 ont été expulsés ces dernières semaines. (....) Saida Benhabyles, présidente du Croissant rouge algérien, organisation humanitaire impliquée dans l'opération, a dénoncé ce qu'elle qualifie de «campagne de dénigrement contre l'Algérie » (...) accuse Benhabyles dans une déclaration à l'agence officielle. (https://www.tsaalgerie.com/expulsion-des-migrants-lappel-des-ong-agace-les-autorites/)

12- Des ONG accusent de nouveau l'Algérie de maltraiter les migrants. Vingt-et-une associations internationales et algériennes, membres d'une obscure Plateforme migration Algérie (PMA), dont Médecins du monde, Caritas ou la Ligue algérienne de défense des droits de l'Homme (LADDH), viennent de ressortir la vieille rengaine sur le sort «tragique » des migrants subsahariens en Algérie. (Algérie patriotique, 02/19/2018)

Nous relevons dans les trois exemples des opérations d'autonymie que nous commentons comme suit :

- Dans l'exemple 10 : les vocables abandon et maltraitance expriment une mise à distance avec les guillemets comme marqueur d'autonymie puisqu'il est question de dénoncer leur contenu accusateur. Cette modalisation en discours second avec laquelle sont employés les vocables maltraitance et abandon renseigne, dans un rapport dialogique, sur l'existence de ce sentiment de mépris envers les réfugiés en Algérie puisque les propos guillemetés sont attribués à d'autres sources. Mais cette même modalisation signale le refus de cautionner une telle réalité pour l'Algérie ;

- Dans l'exemple 11, Saïda Benhabyles, présidente du Croissant rouge algérien, s'insurge et dénonce fermement les accusations des ONG contre l'Algérie. Elle introduit ce discours qu'elle refuse, avec la qualification campagne de dénigrement contre l'Algérie au moyen d'une modalisation autonymique pour signaler la non-coïncidence entre les mots et les choses (Authier-Revuz, 1995) pour indiquer que le fragment employé, en l'occurrence par ceux qui accusent l'Algérie, ne correspond pas à la réalité. Ce dialogisme interdiscursif ${ }^{12}$ nous renseigne sur le caractère polémique de cette partie de discours guillemetée que l'énonciateur refuse (accuse) en tant que représentant officiel du dossier des réfugiés.

- Dans l'exemple 12 : Les guillemets accompagnant l'adjectif tragique traduisent un dire à distance qui vient d'une autre source énonciative et renvoie ainsi à un extérieur discursif. C'est une hétérogénéité énonciative montrée (Authier-Revuz, 1995) qui permet à l'énonciateur, en l'occurrence le journaliste du journal Algérie Patriotique, de se distancer des propos tenus par ces différentes instances pour exprimer son refus face à ces accusations. 
Dénominations Polémiques, Mépris et contre Mépris dans les Discours Produits entre 2017-2019 sur les Réfugiés en Algérie

\subsection{Quand refuser c'est prendre position}

Dans les exemples analysés dans le titre précédent, nous remarquons l'utilisation des verbes accuser et maltraiter pour dénoncer ce discours accusateur dont est victime l'Algérie. En effet, la condamnation de l'acte d'accuser est faite à l'aide d'un acte de langage performatif explicite (dénoncer) qui délégitime cette condamnation dans le discours de Benhabyles (exemple 11) et qui résonne en écho, dans un rapport conflictuel, avec le discours des ONG qui accuse l'Algérie de mépriser les réfugiés. Les verbes accuser et maltraiter (exemple 12) maintiennent ce rejet de l'idée selon laquelle l'Algérie aurait réservé un sort tragique aux réfugiés. Le discours officiellement tenu en/par l'Algérie concernant la question des réfugiés est celui de défendre leur intérêt et de les protéger. Pour étayer cette position, nous proposons, à titre d'exemple, quelques passages de ce discours défenseur des réfugiés en Algérie tenu par la présidente du Croissant rouge algérien lors d'une interview.

«Lors de son intervention, elle [Benhabyles] s'en est, par ailleurs, pris à des internautes qui s'en sont pris avec violence à la population de migrants, dénonçant à travers leur comportement, un chauvinisme et un racisme exacerbés ». (Propos rapportés par la radio algérienne, chaine 3, 27/6/2017 : https://www.radioalgerie.dz/news/fr/article/20170627/115593.html

Sur les actions engagées par l'Algérie, elle déclare :

«(...) l'Algérie a toujours été une terre d'asile (...) c'est dans la tradition (...) l'Algérie a pris ses responsabilités, elle assure toutes les actions humanitaires, (...), pour la scolarisation, nous sommes le seul pays qui encourage cela (...)

Elle s'insurge contre les propos accusateurs :

Quand on a vu ces attaques par certains réseaux sociaux, on se demande si ceux qui sont à l'origine de ces déclarations connaissent leur culture, connaissent leurs repères, connaissent leur religion (...) on n'a pas le droit de tenir de tels propos »

(Extrais de l'intervention de Benhabyles, radio chaine 3, 27/6/2017: https://www. radioalgerie.dz/news/ fr/article/20170627/115593.html

L'énonciatrice s'appuie sur le conscient collectif et utilise les valeurs de la religion comme argument d'autorité, ce qui crée cet espace de riposte et de conflit entre elle et ses interlocuteurs. Par cette stratégie légitimation (Charaudeau, 1995), elle essaie de catégoriser les énonciateurs du discours accusateur en les excluant de la catégorie préétablie (des Algériens musulmans). Elle attribue les accusations aux instances sociales, en l'occurrence les internautes, mais pas aux instances officielles. Les discours tenus par les Autorités algériennes et les actions entreprises par l'Etat algérien vont à l'encontre de ces discours accusateurs.

Il est clair que même si l'Algérie a fait l'objet de plusieurs accusations de la part des ONG concernant le traitement dénigrant réservé aux réfugiés, le discours de la réponse se construit avec les propos tenus par des énonciateurs possédant une légitimé énonciative de par leur position institutionnelle (Président du 
Comité interministériel responsable des problèmes migratoires, Présidente du Croissant rouge algérien) pouvant avancer des arguments pour déconstruire cette image sombre de l'Algérie.

Ces exemples qui dénoncent les accusations qui auraient parlé de mépris envers les réfugiés, attestent d'un interdiscours: le discours tenu par l'Algérie qui conteste un autre discours accusateur déjà existant. Selon ces énonciateurs, les ONG accusatrices de l'Algérie auraient une connaissance partielle de la situation et ne citeraient pas les actions positives que ce pays entreprend envers les réfugiés.

\section{Conclusion}

Les champs lexicaux déployés pour parler des réfugiés, les dénominations polémiques ainsi que la mise en mots d'un discours de réponse fondé sur le refus des accusations présupposent l'existence de mépris envers les réfugiés. Il montre que le mépris concernant un objet social, en l'occurrence ici une catégorie de personnes vulnérables, se traduit à travers le réseau de qualifications et de catégorisations qui sous-tendent la description déployée pour les réfugiés. Le discours polémique des ONG est à vocation évaluative des actions et des comportements de l'Algérie envers les réfugiés ce qui explique le recours aux axiologiques dévalorisants étudiés.

Notre étude s'est basée sur le mépris ressenti et exprimé non pas par les réfugiés eux-mêmes mais à travers le discours rapporté par les ONG, fondé sur l'accusation d'un comportement qui est jugé méprisant. Ce qui peut être déduit de ces analyses, c'est que l'acte du mépris peut être la résultante d'un autre acte de langage direct tel que l'accusation ou la condamnation. Le mépris n'est pas un sentiment à détecter à travers les vocables utilisés mais c'est un processus, c'est un acte qui se construit dans le discours. 
Dénominations Polémiques, Mépris et contre Mépris dans les Discours

Produits entre 2017-2019 sur les Réfugiés en Algérie

$$
\text { تسميات جدلية، ازدراء وضد الازدراء في الخطاب الملقى بين 2017-2019 حول }
$$

$$
\text { كميلة أولبسير-أوكيل }
$$

مختبر اللغويات والتعلم الاجتماعي لتعدد اللغات (LISODIP)، الجزائر

$$
\begin{aligned}
& \text { الملخص } \\
& \text { في هذا المقال، نود أن ندرس تعبير الازدراء الملاحظ في الخطاب الإعلاهي المتعلق باللاجئين في الجزائر. نود أن نبين } \\
& \text { كيف يتهم الخطاب الصادر عن المنظمات غير الحكومية والجمعيات الدوليّة الأخرى الجزائر بازدراء اللاجئين. نريد أيضاً دراسة } \\
& \text { كيف يصيغ هذا البلد رده على اتهامات احتقار اللاجئين. هذه الفئة موصومة بشدة وتعيش في ظروف محفوفة بالمخاطر. } \\
& \text { ستحاول دراستنا تحديد ما يمكن أن يسمى بازدراء لهؤلاء الأشخاص. } \\
& \text { الكلمات المفتاحية: الازدراء، الفعل الكلامي، الخطاب الإعلامي، تحليل الخطاب، الجدل. }
\end{aligned}
$$




\section{Noted de fin}

${ }^{1}$ Des témoignages recensés sur le site de Amnesty Algérie : https://www.amnestyalgerie.org/refugiesmigrants-et-demandeurs-dasile/ ou encore sur les réseaux sociaux (ex. la page «Les réfugiés africains d'Algérie » sur Facebook).

${ }^{2}$ Disponible à l'adresse : http://www.ofii.fr/IMG/pdf/L\%E2\%80\%99accueil\%20du\%20demandeur\%20d\%E2\%80\%99asile/C onvention_de_Geneve.pdf

${ }^{3}$ Extrait du texte de la Constitution algérienne, disponible à l'adresse : http://www.conseilconstitutionnel.dz/index.php/fr/1996/2016

${ }^{4}$ Ibid.

5 Il s'agit, entre autres, de Amnesty International, du Human Rights Watch, du Haut-Commissariat des Nations unies aux droits de l'homme, de l'association Médecins du monde, de l'association Caritas et de la Ligue algérienne de défense des droits de l'homme (LADDH).

${ }^{6}$ Cf. Le rapport de 1'UNHCR de 2012 : https://www.unhcr.org/fr/51efd15d0.pdf

${ }^{7}$ Les différents titres des sources du corpus sont présentés dans la section «Sources du corpus » en bibliographie.

${ }^{8}$ Notamment le hashtag « Non aux Africains en Algérie ».

${ }^{9}$ Cet adjectif en arabe algérien est tiré de l'adjectif khal, « noir » en français.

${ }^{10}$ Ce terme entre dans l'arabe syrien à la suite de l'intervention syrienne au Liban en 1976. Dans le contexte de 2011 et dans le cadre du conflit syrien, ce terme désigne, dans les médias, les milices irrégulières employées par le régime de Béchar EL-Assad pour réprimer l'opposition. Ce sont des jeunes marginaux et mafieux, généralement de la minorité alaouite, responsables de pillages et crimes. (Syrie : les chabiha, ou miliciens pro-régime, 2017:

https://www.ofpra.gouv.fr/sites/default/files/atoms/files/59_syr_les_chabbiha.pdf

${ }^{11}$ Nous avons déjà analysé ce syntagme et son fonctionnement formulaire dans les discours circulant en ligne dans l'espace social algérien (Oulebsir, 2018).

${ }^{12}$ Il s'agit d'un type de dialogisme qui renvoie au fait que « le locuteur, dans sa saisie d'un objet, rencontre les discours précédemment tenus par d'autres sur ce même objet, discours avec lesquels il ne peut manquer d'entrer en interaction »(Bres et Nowakowska, 2001, en ligne). 
Dénominations Polémiques, Mépris et contre Mépris dans les Discours

Produits entre 2017-2019 sur les Réfugiés en Algérie

\section{Références}

Anscombre, Jean-Claude. 1977. La problématique de l'illocutoire dérivé. Langage et société 2: 61-124.

Auger, Nathalie. Fracchiolla, Béatrice. Moïse, Claudine. et Schultz-Romain, Christina. 2008. De la violence verbale, pour une sociolinguistique des discours et des interactions », J. Durand, B. Habert,

B. Laks (dir.) Actes du Congrès Mondial de Linguistique Française (631-643). Paris: Institut de linguistique française. <http://www.linguistiquefrancaise.org>

Austin, John Langshaw. 1970. Quand dire, c'est faire, (G Lane, trad.fr.1962, How to do Things with Words, Oxford), Paris: Seuil.

Authier-Revuz, Jacqueline. 1995. Ces Mots Qui ne Vont pas de Soi. Boucles Réflexives et NonCö̈ncidences du Dire. Paris: Larousse.

Authier-Revuz, Jacqueline. 1992. Repères dans le Champ du Discours Rapporté. L'Information Grammaticale 55: 38-42. Doi: 10.3406/igram.1992.3186.

Bernard Barbeau Geneviève, Moïse Claudine. 2020. Le mépris en discours, Lidil 61. http://journals.openedition.org/lidil/7264.

Bres, Jacques. Nowakowska, Alexandra. 2001. Dis-moi avec qui tu « dialogues », je te dirai qui tu es... De la pertinence de la notion de dialogisme pour l'analyse du discours. Marges Linguistiques, M.L.M.S. http://www.marges-linguistiques.com. ffhal-00438508f.

Calabrese, Laura. 2015. Islamophobie. Une analyse des Dynamiques de la Nomination dans les Commentaires des Lecteurs. Langue française 188: 91-104.

Charaudeau, Patrick. 1995. Ce que communiquer veut dire. Revue des Sciences humaines 51. URL: http://www.patrick-charaudeau.com/Ce-que-communiquer-veut-dire.html.

Fanon Frantz. 1961. Les damnés de la terre, Paris: Maspéro.

Garric Nathalie., Longhi Julien. 2012. L'analyse de corpus face à l'hétérogénéité des données: d'une difficulté méthodologique à une nécessité épistémologique. Langages 187. https://www.cairn. info/revue-langages-2012-3-page-3.htm.

Kerbrat-Orecchioni, Catherine. 1980. L'énonciation. de la Subjectivité dans le Langage, Paris: Armand Colin.

Kerbrat-Orecchioni, Catherine. 2005. Les Actes de Langage dans le Discours. Théorie et Fonctionnement, Paris: Armand Colin.

Krieg-Planque, Alice. 2003. Purification Ethnique: une formule et son histoire, Paris, France: CNRS édition.

Krieg-Planque, Alice. 2009. La notion de Formule en Analyse du Discours. Cadre Théorique et Méthodologique, PU de Franche-Comté.

Laforest, Marty. et Claudine Moïse. 2013. Entre reproche et insulte, comment définir les actes de condamnation? », B. Fracchiolla, C. Moïse, C. Romain, et N. Auger Violences verbales. Analyses, enjeux et perspectives, pp. 85-105. Rennes: Presses Universitaires de Rennes.

Larguèche, Évelyne. 2011. Adresse Indirecte et Injure? Cahiers de Littérature Orale 70: 139-156. DOI: 10.4000/clo.1297. 
Longhi, Julien. 2012. Types de Discours, Formes Textuelles et Normes Sémantiques: Expression et Doxa Dans un Corpus de Données Hétérogènes. Langages 187: 41-58. DOI: 10.3917/lang.187.0041.

Maingueneau Dominique. 2014. Discours et Analyse Du Discours. Introduction, Paris: Armand Colin.

Maingueneau Dominique. 2012. Analyser les textes de communication, nouvelle édition revue et argumentée, Paris: Armand Colin.

Moïse, Claudine. et Christina Romain. 2011. Violence verbale et Listes de Discussions: Les Argumentations Polémiques. Cahiers de l'institut de Linguistique de Louvain, Revue de Sociolinguistique et de Sociologie de la Langue Française 36 (2): 113-132. https://hal.archivesouvertes.fr/hal-01491730.

Moïse, Claudine. 2012. Argumentation, confrontation et Violence Verbale Fulgurante. Argumentation et Analyse du Discours 8. http://journals.openedition.org/aad/1260.

Oulebsir, Kamila. 2018. Autour des Vocables Migrants et Réfugiés: Émergence de Quelques Formules à Partir d'une Situation de Crise. Langues, Discours et Inter Cultures 2: 50-62.

Ukhova, Polina. 2020. De l'Expression du Mépris Au Marquage d'une Solidarité: L'Emploi Ontotypique de Cassos, Beauf, Bolos, Crevard, Kéké et Kikoo dans les Discours Spontanés entre Jeunes. Lidil 61. http://journals.openedition.org/lidil/7274.

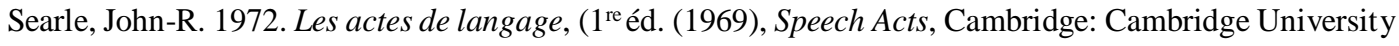
Press), Paris: Hermann.

\section{Sites}

La Convention de Genève, (1951).

http://www.ofii.fr/IMG/pdf/L\%E2\%80\%99accueil\%20du\%20demandeur\%20d\%E2\%80\%99asile/Conve ntion_de_Geneve.pdf> (consulté 05/01/2020).

La Constitution algérienne, (2016). http://www.conseil-constitutionnel.dz/index.php/fr/1996/2016 (consulté 05/01/2020).

Intervention de $\mathrm{S}$. Benhabyles à la chaine 3:

https://www.radioalgerie.dz/news/fr/article/20170627/115593.html

Syrie: les chabiha, ou miliciens pro-régime: 2017, France: DIDR, OFPRA. https://www. ofpra.gouv.fr/sites/default/files/atom s/files/59_syr_les_chabbiha.pdf).

\section{Sources du corpus}

Discours de presse: la presse algérienne d'expression française: Liberté, El Watan, Algérie patriotique, Le Matin d'Algérie.

Articles publiés sur des sites d'information: APS-DZ, TSA, radioalgerie.dz, www.infomigrants.net, mondafrique.com, observalgerie.com, agenceafrique.com.

Articles publiés dans la presse étrangère: Le Monde, Jeune Afrique, Le point.fr. 